\title{
Detecting Observability Problems in Distributed Testing
}

\author{
Jessica Chen ${ }^{1}$ and Hasan $\mathrm{Ural}^{2}$ \\ ${ }^{1}$ School of Computer Science, University of Windsor, \\ Windsor, Ontario, Canada N9B 3P4 \\ xjchen@uwindsor.ca \\ ${ }^{2}$ School of Information Technology and Engineering, \\ University of Ottawa, \\ Ottawa, Ontario, Canada K1N 6N5 \\ uralesite.ottawa.ca
}

\begin{abstract}
Application of a test or checking sequence in a distributed test architecture often requires the use of external coordination message exchanges among multiple remote testers for eluding potential controllability and observability problems. Recent literature reports on conditions on a given finite state machine (FSM) under which controllability and observability problems can be overcome without using external coordination messages. However, these conditions do not guarantee that any test/checking sequence constructed from such FSMs are free from controllability and observability problems. For a given test or checking sequence, this paper investigates whether it is possible to eliminate the need for external coordination messages and proposes algorithms to identify or construct subsequences either within the given sequence or as an extension to the given sequence, respectively.
\end{abstract}

Keywords: Finite state machine, testing, distributed test architecture, observability, controllability.

\section{Introduction}

In a distributed test architecture, there is one tester at each interface/port of the system under test (SUT) $N$. These testers participate in applying a given test sequence [1,15, 16 or checking sequence [7, 9, 11, 19, which is a sequence of input/output pairs, constructed from the specification $M$ of the SUT $N$. The use of multiple remote testers in a distributed architecture brings out the possibility of controllability and observability problems during the application of a test or checking sequence. A controllability problem arises when a tester is required to send the current input and because it did not send the previous input and did not receive the previous output it cannot determine when to send the input. An observability problem arises when a tester is expecting an output in response to either a previous input or the current input and because it is not the 
sender of the current input, it cannot determine when to start and stop waiting for the output.

These problems and their solutions have been studied in the context where $M$ is a Finite State Machine (FSM) and $N$ is a state-based system whose externally observable behavior can also be represented by an FSM. Much of the previous work has been focused on automatically generating test or checking sequences from FSMs that causes no controllability or observability problems during its application in a distributed test architecture (see, for example, [2, 6, 8, 10, 13, 17, 18, 20] ). For some FSMs, there have been test/checking sequences in which the coordination among testers can be achieved indirectly via their interactions with $N$ [14,16. For some others, it may be necessary for testers to communicate directly by exchanging external coordination messages among themselves over a dedicated channel for overcoming the controllability and observability problems encountered during the application of the test/checking sequence [2, 3, 17]. Using external coordination messages introduces delays and the necessity to set up a dedicated communications channel among testers. Thus, the emphasis of the recent work is to minimize the use of external coordination message exchanges among testers [3, 10] or to identify conditions on a given FSM $M$ under which controllability and observability problems can be overcome without using external coordination messages [4,5].

Such conditions lead to the algorithms for identifying paths within a given FSM $M$ that provide evidence for the possibility of eliminating the controllability and observability problems [4,5]. 4] gives conditions on $M$ so that each transition involved in an observability problem can be independently verified at port $p$. By verified at port $p$, it is meant that one can conclude that the output of this transition at port $p$ is correct if one observes the correct output sequence on a certain path within $M$. By independently, it is meant that the above conclusion regarding the output at port $p$ for a transition does not rely on the correctness of any other transitions. Since the notion of independence may not be required in some cases, the above condition on $M$ can be weakened in these cases. [5] gives an algorithm that determines whether $M$ satisfies this weaker condition and when it does so, identifies paths within $M$ that check the output of the transitions.

In this paper, we assume that the given FSM $M$ satisfies the condition in [5]. Then, we pose the following problem and solve it in a restricted setting: Given an FSM $M$ and a synchronizable test or checking sequence $\tau_{0}$ starting at the initial state of $M$, extend $\tau_{0}$ with minimal number of subsequences to form a synchronizable test or checking sequence $\tau^{*}$ such that the detectability of the observability problems in $\tau_{0}$ is guaranteed without using external coordination messages exchanged among remote testers.

The rest of the paper is organized as follows. Section 2 introduces the preliminary terminology. Section 3 gives a formal definition of the general problem and defines a restricted version of this problem. Section 4 presents our solution. Section 5 concludes the paper with our final remarks. 


\section{An $n$-Port FSM and Directed Graphs}

An $n$-port Finite State Machine $M$ (called henceforth an FSM $M$ ) is defined as $M=\left(S, I, O, \delta, \lambda, s_{0}\right)$ where $S$ is a finite set of states; $s_{0} \in S$ is the initial state; $I=\bigcup_{i=1}^{n} I_{i}$, where $I_{i}$ is the set of input symbols of port $i$, and $I_{i} \cap I_{j}=\emptyset$ for $i, j \in[1, n], i \neq j ; O=\prod_{i=1}^{n}\left(O_{i} \cup\{-\}\right)$, where $O_{i}$ is the set of output symbols of port $i$, and - means null output; $\delta$ is the transition function that maps $S \times I$ to $S$; and $\lambda$ is the output function that maps $S \times I$ to $O$. Each $y \in O$ is a vector of outputs, i.e., $y=\left\langle o_{1}, o_{2}, \ldots, o_{n}\right\rangle$ where $o_{i} \in O_{i} \cup\{-\}$ for $i \in[1, n]$. A transition of an FSM $M$ is a triple $t=\left(s_{1}, s_{2}, x / y\right)$, where $s_{1}, s_{2} \in S, x \in I$, and $y \in O$ such that $\delta\left(s_{1}, x\right)=s_{2}, \lambda\left(s_{1}, x\right)=y . s_{1}$ and $s_{2}$ are called the starting state and the ending state of $t$ respectively. The input/output pair $x / y$ is called the label of $t . p \in[1, n]$ will denote a port and we use $\left.y\right|_{p}$ or $\left.t\right|_{p}$ to denote the output at $p$ in output vector $y$ or in transition $t$ respectively. We use $\mathcal{T}$ to denote the set of all transitions in $M$.

A path $\rho=t_{1} t_{2} \ldots t_{k}(k \geq 0)$ is a finite sequence of transitions such that for $k \geq 2$, the ending state of $t_{i}$ is the starting state of $t_{i+1}$ for all $i \in[1, k-1]$. We say $t$ is contained in (or simply in) $\rho$ if $t$ is a transition along path $\rho$. When the ending state of the last transition of path $\rho_{1}$ is the starting state of the first transition of path $\rho_{2}$, we use $\rho_{1} \rho_{2}$ to denote the concatenation of $\rho_{1}$ and $\rho_{2}$. The label of a path $\left(s_{1}, s_{2}, x_{1} / y_{1}\right)\left(s_{2}, s_{3}, x_{2} / y_{2}\right) \ldots\left(s_{k}, s_{k+1}, x_{k} / y_{k}\right)(k \geq 1)$ is the sequence of input/output pairs $x_{1} / y_{1} x_{2} / y_{2} \ldots x_{k} / y_{k}$ which is an input/output sequence.

When $\rho$ is non-empty, we use $\operatorname{first}(\rho)$ and $\operatorname{last}(\rho)$ to denote the first and last transitions of path $\rho$ respectively and $\operatorname{pre}(\rho)$ to denote the path obtained from $\rho$ by removing its last transition.

Given an FSM $M$ and a path $t_{1} t_{2} \ldots t_{k}(k>1)$ of $M$ with label $x_{1} / y_{1}$ $x_{2} / y_{2} \ldots x_{k} / y_{k}$, a controllability (also called synchronization) problem occurs when, in the labels $x_{i} / y_{i}$ and $x_{i+1} / y_{i+1}$ of two consecutive transitions, there exists $p \in[1, n]$ such that $x_{i+1} \in I_{p}, x_{i} \notin I_{p},\left.y_{i}\right|_{p}=-(i \in[1, k-1])$. If this controllability problem occurs then the tester at $p$ does not know when to send $x_{i+1}$ and the test/checking sequence cannot be applied. Consecutive transitions $t_{i}$ and $t_{i+1}$ form a synchronizable pair of transitions if $t_{i+1}$ can follow $t_{i}$ without causing a synchronization problem. Any path in which every pair of consecutive transitions is synchronizable is called a synchronizable path. An input/output sequence is synchronizable if it is the label of a synchronizable path.

We assume that for every pair of transitions $\left(t, t^{\prime}\right)$ there is a synchronizable path that starts with $t$ and ends with $t^{\prime}$. If this condition holds, then the FSM is called intrinsically synchronizable.

Suppose that we are given an FSM $M$ and a synchronizable path $\rho=$ $t_{1} t_{2} \ldots t_{k}$ of $M$ with label $x_{1} / y_{1} x_{2} / y_{2} \ldots x_{k} / y_{k}$. An output shift fault in an implementation $N$ of $M$ exists if one of the following holds for some $1 \leq i<j \leq k$ :

a) For some $p \in[1, n]$ and $o \in O_{p},\left.y_{i}\right|_{p}=o$ in $M$ and for all $i<l \leq j,\left.y_{l}\right|_{p}=-$ in $M$ whereas for all $i \leq l<j, N$ produces output - at $p$ in response to $x_{l}$ after $x_{1} \ldots x_{l-1}$, and $N$ produces output $o$ at $p$ in response to $x_{j}$ after $x_{1} \ldots x_{j-1}$. 
b) For some $p \in[1, n]$ and $o \in O_{p},\left.y_{j}\right|_{p}=o$ in $M$ and for all $i \leq l<j,\left.y_{l}\right|_{p}=-$ in $M$ whereas for all $i<l \leq j, N$ produces output - at $p$ in response to $x_{l}$ after $x_{1} \ldots x_{l-1}$, and $N$ produces output $o$ at $p$ in response to $x_{i}$ after $x_{1} \ldots x_{i-1}$.

In a) the output $o$ shifts from being produced in response to $x_{i}$ to being produced in response to $x_{j}$ and the shift is from $t_{i}$ to $t_{j}$ (i.e., a forward shift). In b) the output $o$ shifts from being produced in response to $x_{j}$ to being produced in response to $x_{i}$ and the shift is from $t_{j}$ to $t_{i}$ (i.e., a backward shift).

An instance of the observability problem manifests itself as a potentially undetectable output shift fault if there is an output shift fault related to $o \in$ $O_{p}$ in two transitions $t_{i}$ and $t_{j}$ in $\rho$ with labels $x_{i} / y_{i}$ and $x_{j} / y_{j}$, such that $x_{i+1} \ldots x_{j} \notin I_{p}$. The tester at $p$ will not be able to detect the faults since it will observe the expected sequence of interactions in response to $x_{i} \ldots x_{j}$. Both $t_{i}$ and $t_{j}$ are said to be involved in the potentially undetectable output shift fault. When $j=i+1$, we also call it potentially undetectable 1-shift output fault.

In the following, $\tau_{0}$ is a given test/checking sequence, which is the label of path $\rho_{0}=t_{1} t_{2} \ldots t_{m}$. We will use $\mathcal{T}_{\rho_{0}, p}$ to denote the set of transitions of $M$ that can be involved in potentially undetectable output shift faults in $\rho_{0}$. Thus $t \in \mathcal{T}_{\rho_{0}, p}$ if there exists a transition $t^{\prime}$ and a synchronizable path $t \rho t^{\prime}$ or $t^{\prime} \rho t$ such that both $t$ and $t^{\prime}$ are involved in a potentially undetectable output shift fault when we apply $\tau_{0}$ to $N$.

Let $t$ be a transition, and $\mathcal{U}$ a set of transitions in $M . \rho$ is an absolute verifying path upon $\mathcal{U}$ for $(t, p)$ if

- $\rho$ is a synchronizable path;

$-t$ is contained in $\operatorname{pre}(\rho)$;

- first $(\rho)$ and last $(\rho)$ and only these two transitions in $\rho$ have input at $p$;

$-t \notin \mathcal{U}$ and for all $t^{\prime}$ contained in $\operatorname{pre}(\rho)$, either $t^{\prime} \in \mathcal{U}$ or $\left.t^{\prime}\right|_{p}=-\left.\Leftrightarrow t\right|_{p}=-[5]$.

Note that given $t$ and $\rho$ we will typically consider a minimal set $\mathcal{U}$ that satisfies the above conditions: if $\left.t^{\prime}\right|_{p}=-\left.\Leftrightarrow t\right|_{p}=-$ then $t^{\prime} \notin \mathcal{U}$.

Suppose that $\mathcal{U}$ is a set of transitions of $M, \mathcal{R} \subseteq \mathcal{U} \times \mathcal{U}$ is a relation, and $\mathcal{P}$ is a function from $\mathcal{U}$ to synchronizable paths of $M$. Let $p$ be any port in $M$. The set $\mathcal{U}$ of transitions is verifiable at $p$ under $\mathcal{R}$ and $\mathcal{P}$ if the following hold [5].

(a) For all $t \in \mathcal{U}, \mathcal{P}(t)$ is an absolute verifying path upon $\left\{t^{\prime} \mid\left(t, t^{\prime}\right) \in \mathcal{R}\right\}$ for $(t, p)$;

(b) $\mathcal{R} \cup\{(t, t) \mid t \in \mathcal{U}\}$ is a partial order.

Where such $\mathcal{R}$ and $\mathcal{P}$ exist we also say that $\mathcal{U}$ is verifiable at $p$.

Let $\mathcal{T}_{p}$ be the set of all transitions involved in some potentially undetectable output shift faults in $M$ at port $p$. In this paper, we assume that $\mathcal{T}_{p}$ is verifiable at $p$ for all $p \in[1, n]$.

A directed graph (digraph) $G$ is defined by a tuple $(V, E)$ in which $V$ is a set of vertices and $E$ is a set of directed edges between the vertices. An edge $e$ from vertex $v_{i}$ to vertex $v_{j}$ is represented by $\left(v_{i}, v_{j}\right)$. A walk is a sequence of pairwise adjacent edges in $G$. A digraph is strongly connected if for any ordered pair of vertices $\left(v_{i}, v_{j}\right)$ there is a walk from $v_{i}$ to $v_{j}$. 


\section{The Problem Definition}

Given a deterministic, minimal, and completely specified FSM $M$ which is intrinsically synchronizable, and a synchronizable test/checking sequence $\tau_{0}$ starting at the initial state of $M$, we consider the problem of constructing a synchronizable test/checking sequence $\tau^{*}$ that can be applied to resolve observability problems in $\tau_{0}$ without using external coordination message exchanges by identifying the subsequences within $\tau_{0}$ or to be appended to $\tau_{0}$.

Clearly, for each $t \in \mathcal{T}_{\rho_{0}, p}$, we should verify its output at port $p$. As we discussed in [5], to verify the output of transition $t$ at port $p$, we can construct an absolute verifying path upon a set $\mathcal{U}$ of transitions whose outputs at $p$ are verified. Such a path $\rho$ has the following properties:

- it is synchronizable;

- we are able to determine the output sequence of $\rho$ at $p$ by applying the label of $\rho$ from the starting state of $\rho$;

- from the correct output sequence of $\rho$ at $p$ we can determine that the output of $t$ at $p$ is correct.

This is because (i) no matter how $\rho$ is concatenated with other subsequences, we can always determine the output sequence produced at $p$ in response to the first $|\operatorname{pre}(\rho)|$ inputs in the label of $\rho$ since this output sequence is immediately preceded and followed by input at $p$; (ii) the condition for all $t^{\prime}$ contained in pre $(\rho)$, either $t^{\prime} \in \mathcal{U}$ or $\left.t^{\prime}\right|_{p}=-\left.\Leftrightarrow t\right|_{p}=-$ allows us to determine the correct output of $(t, p)$ from the correct output sequence of $\rho$ at $p$ (Proposition 2 in [5]).

Thus, to verify the outputs of the transitions in $\mathcal{T}_{\rho_{0}, p}$ at port $p$, we search for an acyclic digraph of transitions such that all transitions in $\mathcal{T}_{\rho_{0}, p}$ are present, and each transition has an absolute verifying path upon a set of transitions that appear as its successors in the digraph. In other words, we search for $\mathcal{R}$ and $\mathcal{P}$ such that set $\mathcal{T}_{\rho_{0}, p}$ of transitions is verifiable at $p$ under $\mathcal{R}$ and $\mathcal{P}$.

It is possible that $\rho_{0}$ contains some absolute verifying paths for transitions in $\mathcal{T}_{\rho_{0}, p}$. Let $Q_{p}$ be the set of all those paths in $\operatorname{codomain}(\mathcal{P})$ but not as subsequences in $\rho_{0} . \tau^{*}$ will be the label of a path $\rho^{*}$ which contains both $\rho_{0}$ and all paths in $Q_{p}$.

Clearly, for efficiency reasons,

- We should maximize the images of $\mathcal{P}$ in $\rho_{0}$. That is, whenever possible, we should define $\mathcal{P}(t)$ as a subsequence in $\rho_{0}$ for any $t \in \mathcal{T}$.

- No path in $Q_{p}$ should appear as a subsequence of another path in $Q_{p}$. This is always true as the absolute verifying paths have input at port $p$ only in its first and last transitions.

- There is no redundant path in $Q_{p}$. An absolute verifying path $\rho$ is redundant in $Q_{p}$ if we can modify $\mathcal{P}$ (and $\mathcal{R}$ correspondingly) by changing the mapping of all transitions whose image is $\rho$ under $\mathcal{P}$ to some other paths in $Q_{p}$ while keeping the property that $\mathcal{T}_{\rho_{0}, p}$ is verifiable at $p$ under the modified definitions of $\mathcal{P}$ and $\mathcal{R}$. Figure 1(a) shows a case where $\left\{t_{1}, t_{2}, t_{3}\right\}$ is verifiable at $p$ under $\mathcal{P}$ and $\mathcal{R}$ where $\mathcal{P}\left(t_{i}\right)=\rho_{i}$ for $i=1,2,3$. Suppose that $\rho_{2}$ is also an absolute verifying path upon $\left\{t_{3}\right\}$ for $\left(t_{1}, p\right)$, then Figure 1(b) shows an 


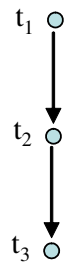

$\rho_{1}$ : an absolute verifying path upon $\left\{t_{2}\right\}$ for $\left(t_{1}, p\right)$ $\rho_{2}$ : an absolute verifying path upon $\left\{t_{3}\right\}$ for $\left(t_{2}, p\right)$ $\rho_{3}$ : an absolute verifying path upon $\phi$ for $\left(t_{3}, p\right)$

(a)

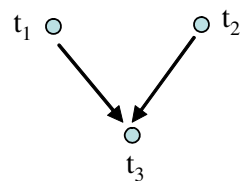

$\rho_{2}$ : an absolute verifying path upon $\left\{t_{3}\right\}$ for $\left(t_{1}, p\right)$ and $\left(t_{2}, p\right)$ $\rho_{3}$ : an absolute verifying path upon $\phi$ for $\left(t_{3}, p\right)$

(b)

Fig. 1. An example of reducing paths in $Q_{p}$

alternative way to verify $\left\{t_{1}, t_{2}, t_{3}\right\}$ which requires less paths in $Q_{p}$ to be considered in constructing $\tau^{*}: \mathcal{P}\left(t_{1}\right)=\mathcal{P}\left(t_{2}\right)=\rho_{2}, \mathcal{P}\left(t_{3}\right)=\rho_{3}$.

\section{Our Proposed Solution}

Now we present our solution to construct $Q_{p}$ and $\tau^{*}$.

\subsection{Identifying Transitions Involved in Observability Problems}

Recall that $\tau_{0}=x_{1} / y_{1} x_{2} / y_{2} \ldots x_{m} / y_{m}$ is a test/checking sequence of $M$ which is the label of a path $\rho_{0}=t_{1} t_{2} \ldots t_{m}$. First we need to calculate $\mathcal{T}_{\rho_{0}, p}$, the set of transitions involved in potentially undetectable output shift faults at port $p$ in $\rho_{0}$, for all $p \in[1, n]$. Figure 2 shows an algorithm for this purpose. It scans $\tau_{0}$ and uses emptyPointer and nonEmptyPointer as auxiliary variables. We do not consider the case when $\left|\tau_{0}\right|=0$ which is meaningless. Suppose we are currently considering $x_{i} / y_{i} \in \tau_{0}$.

emptyPointer is the minimal index of the transitions in $\tau_{0}$ such that

$-\forall k \in[$ emptyPointer $+1, i-1] . x_{k} \notin I_{p}$ and

$-\forall k \in[$ emptyPointer, $i-1] .\left.y_{k}\right|_{p}=-$

nonEmptyPointer is the index of the transitions in $\tau_{0}$ such that

$-\left.y_{k}\right|_{p} \neq-$ for $k=$ nonEmptyPointer and

- $\forall k \in[$ nonEmptyPointer $+1, i-1] .\left.x_{k} \notin I_{p} \wedge y_{k}\right|_{p}=-$

If neither emptyPointer nor nonEmptyPointer is null, then for all $k \in[$ nonEmptyPointer, $i-1], t_{k}$ is involved in a potentially undetectable forward output shift fault. Furthermore, in the case $x_{i} \notin I_{p}$ and $\left.y_{i}\right|_{p}=-, t_{i}$ is also involved in a potentially undetectable forward output shift fault.

If emptyPointer is not null, no matter whether nonEmptyPointer is null or not, $t_{k}$ is involved in a potentially undetectable backward output shift fault for all $k \in[$ emptyPointer, $i]$ when $x_{i} \notin I_{p}$ and $\left.y_{i}\right|_{p} \neq-$. 
1: input: an FSM $M$, a port $p$, a test/checking sequence $\tau_{0}=x_{1} / y_{1} x_{2} / y_{2} \ldots x_{m} / y_{m}$ of $M$

2: output: $\mathcal{T}_{\rho_{0}, p}$

3: nonEmptyPointer $:=$ null

4: emptyPointer $:=$ null

5: $i:=1$

6: while $i<m$ do

7: $\quad$ if $x_{i} \notin I_{p}$ then

8: $\quad$ if $\left.y_{i}\right|_{p} \neq-$ then

9: $\quad$ if emptyPointer $\neq$ null $\wedge$ nonEmptyPointer $\neq$ null then

10: $\quad$ add $t_{n o n E m p t y P o i n t e r}, \ldots, t_{i}$ to $\mathcal{T}_{\rho_{0}, p}$

11: $\quad$ end if

12: $\quad$ if emptyPointer $\neq$ null $\wedge$ nonEmptyPointer $=$ null then

13:

14:

15:

16 :

17:

18:

19:

20:

21:

22:

36: if emptyPointer $\neq$ null $\wedge$ nonEmptyPointer $\neq$ null then

37:

38: end if

39: if emptyPointer $\neq$ null $\wedge$ nonEmptyPointer $=$ null then

40: $\quad$ if $x_{m} \notin I_{p}$ then

41: $\quad$ add $t_{\text {emptyPointer }}, \ldots, t_{m}$ to $\mathcal{T}_{\rho_{0}, p}$

42: else add $t_{\text {emptyPointer }}, \ldots, t_{i}$ to $\mathcal{T}_{\rho_{0}, p}$ end if nonEmptyPointer $:=i$

else

if nonEmptyPointer $=i-1$ then emptyPointer $=i$

end if

end if

else

if emptyPointer $\neq$ null $\wedge$ nonEmptyPointer $\neq$ null then add $t_{n o n E m p t y P o i n t e r}, \ldots, t_{i-1}$ to $\mathcal{T}_{\rho_{0}, p}$

end if

if $\left.y_{i}\right|_{p} \neq-$ then

nonEmptyPointer $:=i$

emptyPointer $:=$ null

else

nonEmptyPointer $:=$ null

emptyPointer $:=i$

end if

end if

add $t_{m}$ to $\mathcal{T}_{\rho_{0}, p}$

end if

45: end if

46: if emptyPointer $=$ null $\wedge$ nonEmptyPointer $\neq$ null then

47: $\quad$ if $x_{m} \notin I_{p}$ and $\left.y_{m}\right|_{p}=-$ then

48: $\quad$ add $t_{m-1}, t_{m}$ to $\mathcal{T}_{\rho_{0}, p}$

49: else

50: $\quad$ add $t_{m}$ to $\mathcal{T}_{\rho_{0}, p}$

51: end if

52: end if

Fig. 2. Algorithm 1: Construction of $\mathcal{T}_{\rho_{0}, p}$ 
Note that some transitions at the end of $\rho_{0}$ that are not involved in any potentially undetectable output shift fault in $\rho_{0}$ may be involved in such faults in the constructed $\rho^{*}$. All these transitions are also added into $\mathcal{T}_{\rho_{0}, p}$ in lines 36-52 which specifically handle the case when $i=m$.

The execution of Algorithm 1 can be done in $\mathcal{O}\left(\left|\tau_{0}\right|\right)$ time.

\subsection{Identifying Verifiable Transitions}

By definition, the transitions in $\mathcal{T}-\mathcal{T}_{\rho_{0}, p}$ all have correct output at $p$. On the other hand, not all transitions in $\mathcal{T}_{\rho_{0}, p}$ need to be verified for its output at $p$ with additional subsequences. This is based on the following two observations:

- A transition in $\mathcal{T}_{\rho_{0}, p}$ may appear in a different place in $\rho_{0}$ where it is not involved in any potentially undetectable output shift faults at $p$ in $\rho_{0}$, and thus its output at $p$ is verified in $\rho_{0}$.

- Given a transition $t \in \mathcal{T}_{\rho_{0}, p}$, there may exist an absolute verifying path upon $\mathcal{T}-\mathcal{T}_{\rho_{0}, p}$ for $(t, p)$ in $\rho_{0}$.

In general, before constructing additional subsequences to be appended to $\tau_{0}$, we would like to find $\mathcal{R}_{0}, \mathcal{P}_{0}$ and $\mathcal{U}_{0} \subset \mathcal{T}_{\rho_{0}, p}$ such that

- $\mathcal{U}_{0}$ is verifiable at $p$ under $\mathcal{R}_{0}$ and $\mathcal{P}_{0}$ in $\rho_{0}$, in the sense that $\mathcal{U}_{0}$ is verifiable at $p$ under $\mathcal{R}_{0}$ and $\mathcal{P}_{0}$, and the paths in $\operatorname{codomain}\left(\mathcal{P}_{0}\right)$ are all in $\rho_{0}$;

$-\mathcal{U}_{0}$ is maximized, in the sense that for any $\mathcal{R}_{0}^{\prime}, \mathcal{P}_{0}^{\prime}$ and $\mathcal{U}_{0}^{\prime}$ such that $\mathcal{U}_{0}^{\prime}$ is verifiable at $p$ under $\mathcal{R}_{0}^{\prime}$ and $\mathcal{P}_{0}^{\prime}$ in $\rho_{0}, \mathcal{U}_{0}^{\prime} \subseteq \mathcal{U}_{0}$.

The following proposition follows directly from the definition.

Proposition 1. Let $\rho$ be a synchronizable path with input at $p$ only in $\operatorname{first}(\rho)$ and last $(\rho)$, and $t \in \operatorname{pre}(\rho)$. Let $D_{t, \rho}$ be the set of transitions in $\operatorname{pre}(\rho)$ such that for any $t^{\prime} \in D_{t, \rho},\left.t^{\prime}\right|_{p}=-\left.\Leftrightarrow t\right|_{p} \neq-$. Then $\rho$ is an absolute verifying path upon $D_{t, \rho}$ for $(t, p)$.

Let $\rho$ be a subsequence in $\rho_{0}$ with input at $p$ both at the beginning and at the end. Based on the above proposition, if the set of all those transitions in $\rho$ with empty output at $p$ is verifiable, then the set of all transitions in $\rho$ is verifiable using $\rho$ as an absolute verifying path. Analogously, if the set of all those transitions in $\rho$ with non-empty output at $p$ is verifiable, then the set of all transitions in $\rho$ is verifiable.

Thus, we can derive from $\rho_{0}$ a set of so-called counter-pairs $\left(L_{1}, L_{2}\right)$ of sets of transitions. Each counter-pair $\left(L_{1}, L_{2}\right)$ corresponds to a potential candidate of absolute verifying path in $\rho_{0}$ that can be used in defining $\mathcal{P}$. It is obtained in this way: for any subsequence $\rho$ of $\rho_{0}$ with input at $p$ both at the beginning and at the end (and no other input at $p$ in it), there is a counter-pair $\left(L_{1}, L_{2}\right)$ where $L_{1}$ contains all transitions in $\operatorname{pre}(\rho)$ with empty output at $p$, and $L_{2}$ contains all transitions in $\operatorname{pre}(\rho)$ with non-empty output at $p$. Such counter-pairs hold the following property: for any set $A$ of transitions in $\mathcal{T}$, the outputs of all transitions in $L_{1}$ are verifiable upon $A$ implies the outputs of all transitions 
1: input: an FSM $M$, a port $p$, a test/checking sequence $\tau_{0}=x_{1} / y_{1} x_{2} / y_{2} \ldots x_{m} / y_{m}$ of $M$, and $\mathcal{T}_{\rho_{0}, p}$

2: output: a set $\mathcal{U}_{0}$ of transitions that is verifiable at $p$ in $\rho_{0}$, and a set $\Theta$ of counterpairs of $p$

3: $\Theta:=\emptyset$

4: Let $r \leq m$, s.t. $x_{r} \in I_{p}$ and $\forall k, 1 \leq k<r, x_{k} \notin I_{p}$

5: while $\exists j . r<j \leq m$ s.t. $x_{j} \in I_{p}$ and $\forall k, r<k<j, x_{k} \notin I_{p}$ do

6: $\quad$ let $j$ be such that $r<j \leq m, x_{j} \in I_{p}$ and $\forall k, r<k<j, x_{k} \notin I_{p}$

7: $\quad$ if $\exists r \leq k<j$ s.t. $t_{k} \in \mathcal{T}_{\rho_{0}, p}$ then

8: $\quad L_{1}:=\emptyset$

9: $\quad L_{2}:=\emptyset$

10: $\quad$ for $k, r \leq k<j$ do

11: $\quad$ if $\left.y_{k}\right|_{p}=-$ then

12: $\quad$ add $t_{k}$ to $L_{1}$

13: else

14: $\quad$ add $t_{k}$ to $L_{2}$

15: end if

16: end for

17: $\quad$ add $\left(L_{1}, L_{2}\right)$ to $\Theta$

18: end if

19: $\quad r=j$

20: end while

21: $\left(\mathcal{U}^{\prime}, \Theta^{\prime}\right):=$ counterPairsUpdate $\left(\mathcal{T}-\mathcal{T}_{\rho_{0}, p}, \Theta\right)$

22: return $\mathcal{U}^{\prime}$ and $\Theta^{\prime}$

Fig. 3. Algorithm 2: Construction of $U_{0}$ and $\Theta$

in $L_{2}$ are verifiable upon $A \cup L_{1}$; and the outputs of all transitions in $L_{2}$ are verifiable upon $A$ implies the outputs of all transitions in $L_{1}$ are verifiable upon $A \cup L_{2}$. Consequently, for any $t \in L_{1}$, the path corresponding to $\left(L_{1}, L_{2}\right)$ can be used as an absolute verifying path upon $\mathcal{U}$ for $(t, p)$ if $L_{2} \subseteq \mathcal{U}$. Conversely, for any $t \in L_{2}$, the path corresponding to $\left(L_{1}, L_{2}\right)$ can be used as an absolute verifying path upon $\mathcal{U}$ for $(t, p)$ if $L_{1} \subseteq \mathcal{U}$.

Figure 3 gives an algorithm to calculate set $\mathcal{U}_{0}$ of transitions whose outputs at $p$ are verifiable in $\rho_{0}$. Set $\Theta$ contains those counter-pairs that correspond to potential candidates of absolute verifying paths. Given a set $\mathcal{U}_{0}$ of transitions that is verifiable at $p$ under $\mathcal{R}_{0}$ and $\mathcal{P}_{0}$ in $\rho_{0}$, we can check if any potential candidate of absolute verifying path can be used to extend $\mathcal{U}_{0}$. This operation is performed in Figure 4. Counter-pairs whose corresponding paths will no more be used during the construction of $\mathcal{R}_{0}$ and $\mathcal{P}_{0}$ are removed from $\Theta$.

Note that if there is no input in $\tau_{0}$ that will be given at port $p$, then we are not able to construct an absolute verifying path for any output at $p$. Since we assume that $\mathcal{T}_{p}$ is verifiable, this implies that $\mathcal{T}_{\rho_{0}, p}=\emptyset$, and thus there is no need for the subsequences to be appended to $\rho_{0}$ for port $p$. Hence we consider there is at least one input at $p$ in $\tau_{0}$.

At the end of Algorithm 2, we have that (i) $\mathcal{U}_{0}$ is verifiable at $p$ under $\mathcal{R}_{0}$ and $\mathcal{P}_{0}$ in $\rho_{0}$, and it is maximized; (ii) all potential absolute verifying paths in $\rho_{0}$ for further use have their correspondence in $\Theta$. 


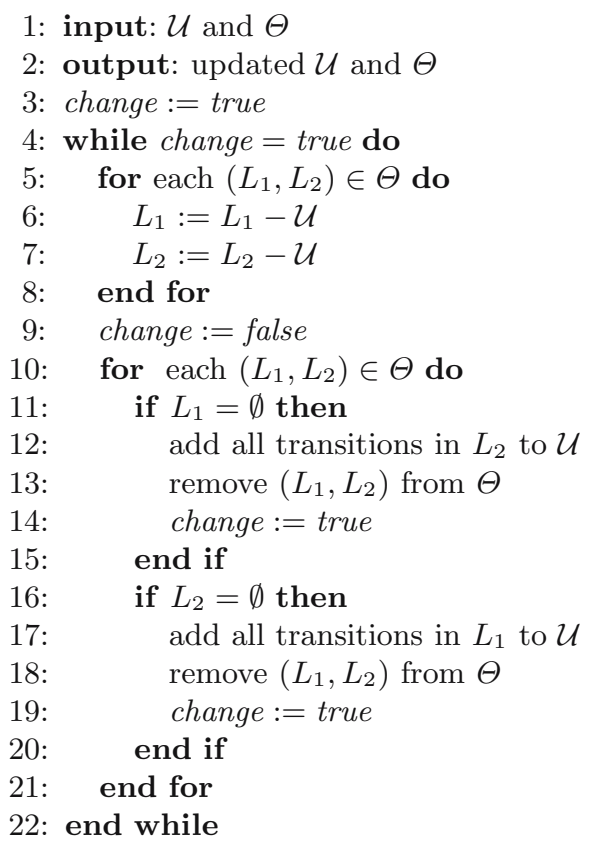

Fig. 4. Procedure of counterPairsUpdate

We know that $\Sigma_{\left(L_{1}, L_{2}\right) \in \Theta}\left(\left|L_{1}\right|+\left|L_{2}\right|\right) \leq\left|\tau_{0}\right|$, and $|\mathcal{U}| \leq|\mathcal{T}|$. So in Figure 4, the first for-loop will be executed maximally $\left|\tau_{0}\right| \times|\mathcal{T}|$ times, and the second forloop will be executed maximally $\left|\tau_{0}\right|$ times. The while-loop each time removes at least one counter-pair from $\Theta$. So in total it takes $\mathcal{O}\left(\left|\tau_{0}\right| \times|\mathcal{T}| \times|\Theta|\right)$ time to perform counterPairsUpdate. Consequently, it takes $\mathcal{O}\left(\left|\tau_{0}\right| \times|\mathcal{T}| \times|\Theta|\right)$ time to run Algorithm 2.

\subsection{Identifying Subsequences to Be Added to $\tau_{0}$}

Given an initial set $\mathcal{U}_{0}$ of transitions that is verifiable at $p$ in $\rho_{0}$, and a set $\Theta$ of counter-pairs corresponding to some potential absolute verifying paths, we define $\mathcal{P}$ and $\mathcal{R}$ such that $\mathcal{T}_{\rho_{0}, p}$ is verifiable at $p$ under $\mathcal{R}$ and $\mathcal{P}$; the images of $\mathcal{P}$ in $\rho_{0}$ is maximized; there is no redundant path in $\mathcal{U}$. This leads to the construction of $Q_{p}$ that we want.

Figure 5 gives an algorithm to construct $Q_{p}$. Here checkset is used to keep the transitions that we may need to construct additional subsequences to verify their output at $p$. Since we assume that $\mathcal{T}_{p}$ is verifiable at port $p, \mathcal{T}_{\rho_{0}, p}-\mathcal{U}$ is also verifiable. So for each iteration of the outer while-loop, we can surely find an absolute verifying path upon $\mathcal{U}$ for some $t \in$ checkset before checkset becomes empty.

Whenever we find an absolute verifying path upon $\mathcal{U}$ for some $t \in$ checkset, we add to $\mathcal{U}$ all transitions in $\operatorname{pre}(\rho)$ such that they have empty output at $p$ if and only if $t$ has empty output at $p$. This is because if $\rho$ is an absolute verifying path upon $\mathcal{U}$ for $(t, p)$, then $\rho$ is an absolute verifying path upon $\mathcal{U}$ for $\left(t^{\prime}, p\right)$ for 


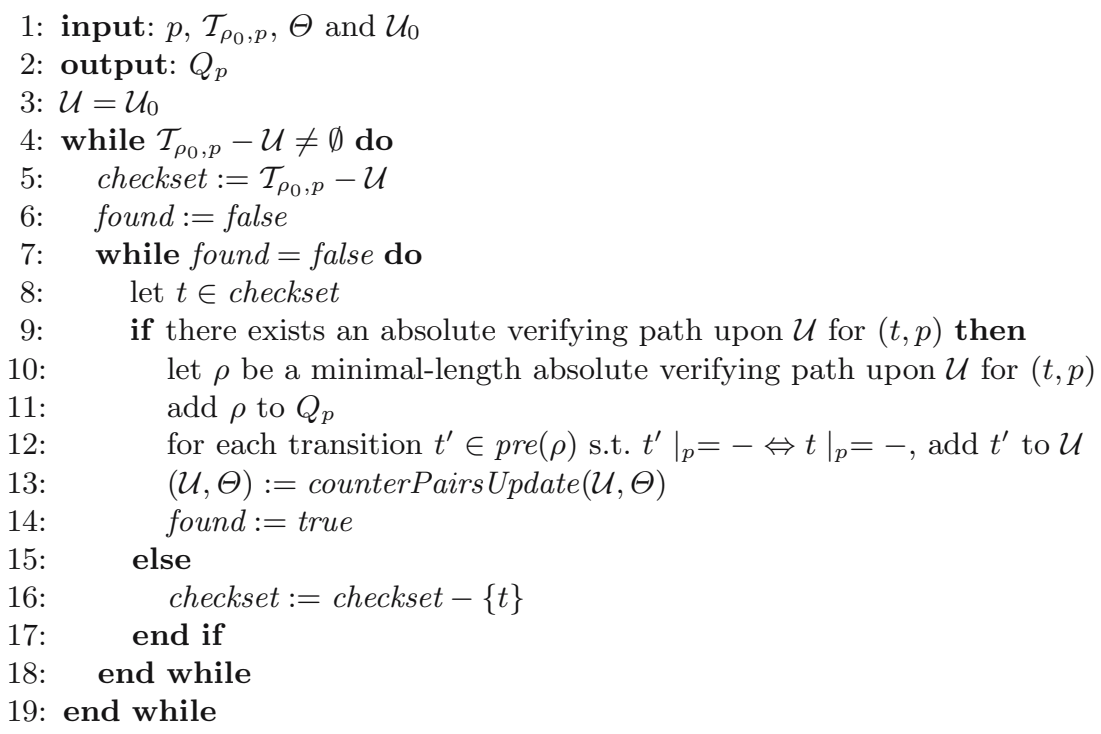

Fig. 5. Algorithm 3: Construction of $Q_{p}$

all $t^{\prime} \in \operatorname{pre}(\rho)$ such that $\left.t^{\prime}\right|_{p}=-\left.\Leftrightarrow t\right|_{p}=-$ (Proposition 1 in [5]). This also guarantees that when we search for an absolute verifying path upon $\mathcal{U}$ for $(t, p)$, we do not need to check whether previously constructed subsequences in $Q_{p}$ can be re-used. Consequently, there is no redundant path in $\mathcal{U}$.

Whenever an additional sequence is constructed and added to $Q_{p}, \mathcal{U}$ is updated. Correspondingly, we call procedure counterPairsUpdate to check if based on the updated $\mathcal{U}$ any potential absolute verifying path in $\rho_{0}$ can be used. As the initial value of $\mathcal{U}$ is from Algorithm 2, this guarantees that for any $\rho \in Q_{p}$, $\rho$ is not a subsequence of $\rho_{0}$. Thus, the images of $\mathcal{P}$ in $\rho_{0}$ is maximized.

¿From [5], we know that if $\rho$ is an absolute verifying path upon $\mathcal{U}$ for $(t, p)$, then when we apply the label of $\rho$ from a state in $N$ similar to the starting state of $\rho$, then we can verify that the output of $t$ at $p$ is correct. So, when we have $\mathcal{T}_{\rho_{0}, p}-\mathcal{U}=\emptyset$ at the end of the algorithm, we know that if we apply $\tau_{0}$ from the initial state of $N$ and apply the label of $\rho$ from a state similar to the starting state of $\rho$ for all $\rho \in Q_{p}$, then we can verify that there is no undetectable output shift faults occurred in applying $\tau_{0}$ to $N$.

To find a minimal-length absolute verifying path upon $\mathcal{U}$ for $(t, p)$, similar as in [5], we can construct $G[t, \mathcal{U}]$ which is obtained from $G$ by removing all edges except those corresponding to a transition $t^{\prime}$ in one of the following cases:

$-t^{\prime}$ has input at $p$;

$-\left.t^{\prime}\right|_{p}=-$ if and only if $\left.t\right|_{p}=-$;

$-t^{\prime} \in \mathcal{U}$

We then use breadth-first search to construct minimal-length synchronizable path in $G[t, \mathcal{U}]$ that starts with input at $p$ and ends with input at $p$. Note that there may exist more than one such path with minimal-length. 
Note also that while more transitions are added to $\mathcal{U}$, there may exist shorter path for a transition whose image under $\mathcal{P}$ was previously added to $Q_{p}$.

Now we turn to the complexity of the algorithm. For each outer while-loop, $\mathcal{U}$ is augmented by at least one transition. So the outer while-loop will be executed at most $v$ times where $v$ is the number of transitions to be verified. For the inner while-loop, we need to check if we can find an absolute verifying path upon $\mathcal{U}$ for some $t \in$ checkset where $\mid$ checkset $\mid \leq v$. This can be realized by trying to construct an absolute verifying path upon $\mathcal{U}$ for each $t$ in checkset until such a path is found. This takes at most $\mid$ checkset $\mid$ times of effort for each attempt. For each attempt to construct an absolute verifying path upon $\mathcal{U}$ for a given transition $t$, it takes $\mathcal{O}(w \times|\mathcal{T}|)$ times where $w$ is the number of states in $M$. In summary, the time complexity of Algorithm 3 is $\mathcal{O}\left(v^{2} \times w \times|\mathcal{T}|\right)$.

\subsection{Adding Subsequences to $\tau_{0}$}

Finally, given $\rho_{0}$ and $Q_{p}$ for each $p$, we need to construct a minimal-length test/checking sequence $\tau^{*}$ so that (i) it is synchronizable; (ii) it starts with $\tau_{0}$ and it contains all the input/output sequences of the paths in $Q_{p}$ for each $p \in[1, n]$. Figure 6 gives such an algorithm. It generates a synchronizable path $\rho^{*}$ and its label $\tau^{*}$.

1: input: $M, \tau_{0}$, and $Q_{p}$ for each $p \in[1, n]$

2: output: test/checking sequence $\tau^{*}$

3: Let $Q=\cup_{p \in[1, n]} Q_{p} \cup\left\{\rho_{0}\right\}$

4: Let graph $G$ contain one vertex $v_{\rho}$ for each path $\rho$ in $Q$

5: for each ordered pair $\left(\rho_{1}, \rho_{2}\right) \in Q$ such that $\rho_{1} \neq \rho_{2}$ do

6: find a shortest path $\rho^{\prime}$ in $M$ such that $\operatorname{last}\left(\rho_{1}\right) \rho^{\prime} \operatorname{first}\left(\rho_{2}\right)$ is a synchronizable path.

7: $\quad$ In $G$, add an edge $e=\left(v_{\rho_{1}}, v_{\rho_{2}}\right)$, with $\left|\rho^{\prime}\right|$ as its weight

8: $\quad$ let $f_{1}(e)=\rho_{1}, f_{2}(e)=\rho_{1} \rho^{\prime}, f_{3}(e)=\rho_{1} \rho^{\prime} \rho_{2}$

9: end for

10: Find a walk $r=e_{1} e_{2} \ldots e_{k}$ in $G$ that visits all vertices at least once with minimal cost, and that $f_{1}\left(e_{1}\right)=\rho_{0}$

11: Let $\rho^{*}=f_{2}\left(e_{1}\right) f_{2}\left(e_{2}\right) \ldots f_{2}\left(e_{k-1}\right) f_{3}\left(e_{k}\right)$

12: Let $\tau^{*}$ be the label of $\rho^{*}$

Fig. 6. Algorithm 4: Addition of elements of $Q_{p}$ to $\rho_{0}$ to form $\rho^{*}$

As we assume that $M$ is intrinsically synchronizable, $G$ is a stronglyconnected digraph. This guarantees the existence of $r$. In general, the time complexity of Algorithm 4 is equivalent to that of finding a travelling saleman tour in a digraph. Efficient heuristics exist for the solution of Travelling Saleman Problem, cf. [12].

Note that $\rho^{*}$ may introduce new observability problems. However, since each path in $Q_{p}$ has input at $p$ in its first and last transitions, a new observability problem cannot happen between a transition in a connecting path, i.e. a path 
used to connect paths in $Q_{p}$, and a transition in an absolute verifying path in $Q_{p}$. It can only happen (i) within a connecting path; (ii) within an absolute verifying path; or (iii) between a transition in $\rho_{0}$ and a transition in a connecting path. The new observability problems occurred in cases (i) and (ii) do not affect the ability of $\tau^{*}$ to verify that there is no undetectable output shift faults when $\tau_{0}$ is applied to $N$. The new observability problems in case (iii) are resolved because we have included into $\mathcal{T}_{\rho_{0}, p}$ all transitions that may possibly get involved in some potentially undetectable output shift fault between a transition in $\rho_{0}$ and a transition in a path concatenated to the end of $\rho_{0}$ (cf. Algorithm 1).

\section{Conclusions and Final Remarks}

We have presented a method for eliminating the use of external coordination message exchanges for resolving observability problems in a given test/checking sequence constructed from an FSM satisfying conditions given in [5]. There are various optimization problems remaining to be solved. First, the existence of multiple minimal-length absolute verifying paths can be used to optimize the total length of $\rho^{*}$. Second, in our solution, the order of generating the subsequences will have an effect on the final set of additional subsequences. It will be interesting to find approaches for eliminating this effect. Third, our solution only considers the subproblem of constructing the subsequences for each port $p$ individually. It remains as an interesting problem to consider the global optimization problem among all ports. Fourth, it will be quite interesting to incorporate some of the algorithms proposed here into a checking sequence construction method to construct a checking sequence in which there are no external coordination message exchanges. It is anticipated that the complexity of the last two optimization problems will be very high.

\section{Acknowledgements}

This work is supported by Natural Sciences and Engineering Research Council (NSERC) of Canada under grant RGPIN 976 and 209774.

\section{References}

1. A. V. Aho, A. T. Dahbura, D. Lee, and M. U. Uyar. An optimization technique for protocol conformance test generation based on UIO sequences and Rural Chinese Postman Tours. In Protocol Specification, Testing, and Verification VIII, pages 75-86, Atlantic City, 1988. Elsevier (North-Holland).

2. S. Boyd and H. Ural. The synchronization problem in protocol testing and its complexity. Information Processing Letters, 40:131-136, 1991.

3. L. Cacciari and O. Rafiq. Controllability and observability in distributed testing. Information and Software Technology, 41:767-780, 1999.

4. J. Chen, R. M. Hierons, and H. Ural. Conditions for resolving observability problems in distributed testing. In 24rd IFIP International Conference on Formal Techniques for Networked and Distributed Systems (FORTE 2004), volume 3235 of LNCS, pages 229-242. Springer-Verlag, 2004. 
5. J. Chen, R. M. Hierons, and H. Ural. Resolving observability problems in distributed test architecture. In 25rd IFIP International Conference on Formal Techniques for Networked and Distributed Systems (FORTE 2005), volume 3731 of LNCS, pages 219-232. Springer-Verlag, 2005.

6. W. Chen and H. Ural. Synchronizable checking sequences based on multiple UIO sequences. IEEE/ACM Transactions on Networking, 3:152-157, 1995.

7. A. Gill. Introduction to the Theory of Finite-State Machines. New York: McGrawHill, 1962.

8. S. Guyot and H. Ural. Synchronizable checking sequences based on UIO sequences. In Proc. of IFIP IWPTS'95, pages 395-407, Evry, France, September 1995.

9. F.C. Hennie. Fault detecting experiments for sequential circuits. In Proc. of Fifth Ann. Symp. Switching Circuit Theory and Logical Design, pages 95-110, Princeton, N.J., 1964.

10. R. M. Hierons. Testing a distributed system: generating minimal synchronised test sequences that detect output-shifting faults. Information and Software Technology, 43(9):551-560, 2001.

11. D. Lee and M. Yannakakis. Principles and methods of testing finite-state machines - a survey. Proceedings of the IEEE, 84(8):1089-1123, 1996.

12. S. Lin and B. W. Kernighan. An effective heuristic algorithm for the travelingsalesman problem. Operations Research, 21(2):498-516, March-April 1973.

13. G. Luo, R. Dssouli, and G. v. Bochmann. Generating synchronizable test sequences based on finite state machine with distributed ports. In The 6th IFIP Workshop on Protocol Test Systems, pages 139-153. Elsevier (North-Holland), 1993.

14. G. Luo, R. Dssouli, G. v. Bochmann, P. Venkataram, and A. Ghedamsi. Test generation with respect to distributed interfaces. Computer Standards and Interfaces, 16:119-132, 1994.

15. K.K. Sabnani and A.T. Dahbura. A protocol test generation procedure. Computer Networks, 15:285-297, 1988.

16. B. Sarikaya and G. v. Bochmann. Synchronization and specification issues in protocol testing. IEEE Transactions on Communications, 32:389-395, April 1984.

17. K.C. Tai and Y.C. Young. Synchronizable test sequences of finite state machines. Computer Networks, 13:1111-1134, 1998.

18. H. Ural and Z. Wang. Synchronizable test sequence generation using UIO sequences. Computer Communications, 16:653-661, 1993.

19. H. Ural, X. Wu, and F. Zhang. On minimizing the lengths of checking sequences. IEEE Transactions on Computers, 46:93-99, 1997.

20. Y.C. Young and K.C. Tai. Observation inaccuracy in conformance testing with multiple testers. In Proc. of IEEE WASET, pages 80-85, 1998. 